\title{
Eternal dilemma of the interplay between coexisting tuberculosis and malignancy: Clinical scenario of coexisting submandibular carcinoma with disseminated tuberculosis: A case report
}

\author{
Manjari Shah, ${ }^{1}$ Nitin Leekha, ${ }^{2}$ Sweety Gupta, ${ }^{3}$ Sowmiya S, ${ }^{4}$ Garima Garg, ${ }^{5}$ Sudarsan De ${ }^{6}$ \\ ${ }^{1} D N B$ Resident, Department of Radiation Oncology, Max Superspeciality Hospital, Vaishali, Ghaziabad, India, ${ }^{2}$ Consultant, Department \\ of Surgical Oncology, Max Superspeciality Hospital, Vaishali, Ghaziabad, India, ${ }^{3}$ Consultant, Department of Radiation Oncology, Max \\ Superspeciality Hospital, Vaishali, Ghaziabad, India, ${ }^{4}$ Senior Resident, Department of Radiation Oncology, Max Superspeciality Hospital, \\ Vaishali, Ghaziabad, India, ${ }^{5}$ Consultant, Department of Pathology, Max Superspeciality Hospital, Vaishali, Ghaziabad, India, ${ }^{6} \mathrm{Head}$ and Senior \\ Consultant, Department of Radiation Oncology, Max Superspeciality Hospital, Vaishali, Ghaziabad, India
}

\section{A B S T R A C T}

\begin{abstract}
Tuberculosis (TB) and malignancy are two lethal diseases that cause significant morbidity and mortality in developing countries like India. Extrapulmonary tuberculosis (TB) constitutes about 15 to 20 per cent of all cases of tuberculosis with lymph nodes being the most common site of involvement. Malignant transformation in pleomorphic adenoma lead to carcinoma ex pleomorphic adenoma (CXPA). CXPA of the salivary glands, however, is relatively uncommon, accounting for $3.6 \%$ of all salivary gland neoplasms and $11.7 \%$ of all malignant salivary gland neoplasms. Here we report a case of carcinoma ex pleomorphic adenoma of submandibular gland which presented as a swelling in submandibular region along with generalized lymphadenopathy which mimicked widespread metastases but later on histologically showed tuberculosis.
\end{abstract}

\section{Access this article online}

Website:

http://nepjol.info/index.php/AJMS

DOI: 10.3126/ajms.v7i3.13634

E-ISSN: 2091-0576

P-ISSN: 2467-9100

Key words: Tuberculosis, Malignancy, Salivary gland, Lymphadenopathy

\section{CASE REPORT}

A 50 year old gentleman presented to the oncology out-patient department with a painless lump in right side of upper neck for last 8 years. The lump progressively increased in size since last 2 months. He also complained of abdominal distension and bilateral lower limb swelling. On examination, ECOG performance score was zero, a $4 \times 5 \mathrm{~cm}$, firm, nontender lump with restricted mobility was located in the right submandibular region. Multiple bilateral cervical (level I-V), supraclavicular and left axillary lymph nodes were palpable with largest being in left axillary region measuring $1 \times 1.5 \mathrm{~cm}$. Per-abdomen examination revealed presence of free fluid, hepatomegaly with normal per-rectal examination.

Fine needle aspiration cytology [FNAC] from right submandibular swelling was done which reported deposits from carcinoma, possibly squamous cell carcinoma. Excision biopsy from left supraclavicular lymph node revealed necrotizing granulomatous lymphadenitis suggestive of tuberculosis. Whole body FDG-PET CT Scan (Figure 1a) was done which showed enhancing nodular mass lesion in right submandibular region (SUV max- 3.31) likely a lymph nodal mass, submandibular salivary gland was not separately visualized, increased FDG uptake seen in multiple cervical, axillary and abdomino-pelvic lymph nodes, and subcentimetric lesions with increased uptake in both lobes of liver, spleen andomentum.FDG avid concentric thickening (Figure 1b) with luminal compromise was seen involving caecum and ileocaecal junction(SUV max-7.10). PET-CT scan findings suggested likely co-existing histologically two different pathologies tuberculosis and lymphoma/carcinoma.

In view of PET-CT scan finding lower gastrointestinal endoscopy was done which was normal. Tru-cut biopsy was repeated from right submandibular region, which 
reported carcinoma. Patient was discussed in tumor board and then was taken up for surgery. Right submandibular gland excision with right modified radical neck dissection along with left cervical lymph node biopsy was done. On frozen section left sided cervical lymph node showed necrotizing granulomatous inflammation. Post-operative histopathology reported extensive areas of squamous cell carcinoma possibly of carcinoma ex pleomorphic adenoma in right submandibular gland (Figure 2a). No perineural invasion or lymphovascular invasion was identified. Tumor was infiltrating into the surrounding fat, however not involving the overlying skin and muscle. Right cervical lymph nodes showed necrotizing granulomatous inflammation (Figure 2b) but none showed malignancy.

Antituberculous (ATT) treatment was started. Now patient is undergoing post-operative adjuvant external beam radiotherapy.

\section{DISCUSSION}

Malignant derivatives of pleomorphic adenoma of salivary gland origin called malignant mixed tumors, can be divided into three different types:

1. CXPA,

2. Carcinosarcoma (true malignant mixed tumor),

3. M-PA [metastasizing pleomorphic adenoma].

CXPA comprises the vast majority of malignant mixed tumors and accounts for $11.7 \%$ of all salivary gland
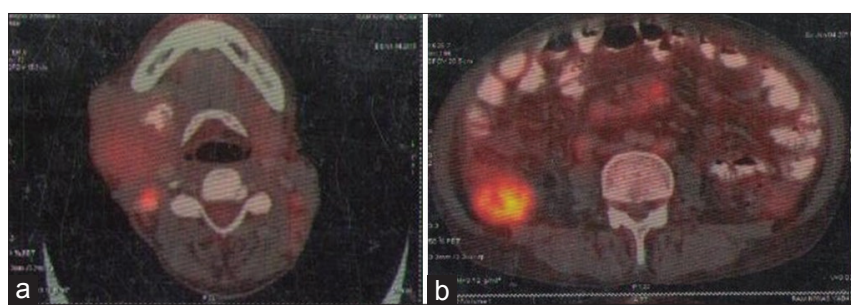

Figure 1: (a) PET CT scan showing enhancing nodular mass lesion in right submandibular region (SUV max- 3.31) likely a lymph nodal mass. (b) FDG avid concentric thickening with luminal compromise was seen involving caecum and ileocaecal junction (SUV max-7.10)

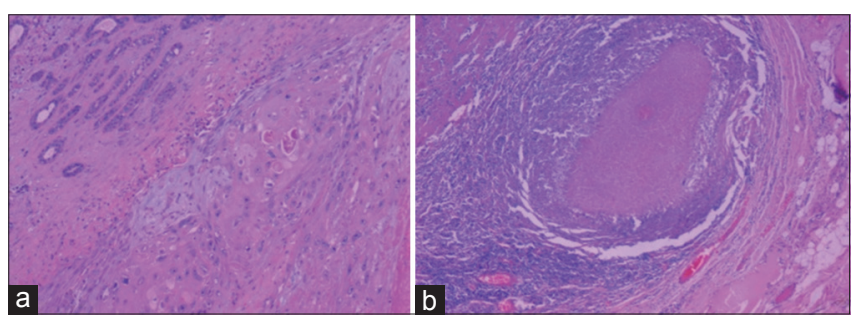

Figure 2: (a) Malignant myoepithelial cells forming glands and cords coexisting with malignant squamous component in a myxoid stroma (H\&E, 200x). (b) Lymph node showing necrotizing granulomatous inflammation (H\&E, 200x) malignancies, whereas the last two types are much rarer. ${ }^{2,3}$ Most CXPAs occur in the parotid gland, and their occurrence in other salivary glands, such as the submandibular gland, is rather rare. ${ }^{4}$ Only less than 7 $\%$ of CXPAs are found in the minor salivary glands. ${ }^{5}$ Coexisting tuberculosis and malignancy poses clinical, radiological difficulties and leads to diagnostic dilemma along with over staging of disease. ${ }^{1}$ It become more challenging to differentiate between the two when on imaging studies such as combined (PET) positron emission tomography and computed tomography (CT) these two pathologies mimic each other and on tissue biopsies tuberculous inflammatory changes and malignant cells co-exist. Kaplan et al, studied 210 cases which had coexistence of TB and malignancies and active tuberculosis complicating the neoplastic disease. ${ }^{6}$ According to Kaplan, tuberculosis was most prevalent in patients with Hodgkin's disease (HD) and lung cancer, whereas it was least prevalent in patients with carcinoma of the colon and bladder. Head and neck cancer prevalence was 51 per 10000 population and most of the time it presented as pulmonary tuberculosis with only one case that presented as disseminated disease. Similarly, Falgas conducted an extensive review of literature regarding the association of these two diseases and highlighted the neglected aspects of this association. ${ }^{7}$ Tuberculosis occurring in Warthin's tumour and pleomorphic adenoma of the salivary gland has been documented in the literature. However in all, the cases numbers are few and both the pathologies occurred concurrently in salivary gland. ${ }^{8}$ It poses diagnostic and therapeutic challenge for the oncologist as there are no pathognomonic symptoms or signs to distinguish both diseases.

\section{CONCLUSION}

Malignancy of submandibular salivary gland and its co-existence with extrapulmonary tuberculosis gives overlapping features. Hence care should be taken during radiological or histopathological examination. In this case submandibular salivary gland carcinoma presented as a lump in right upper neck and generalized lymphadenopathy with increased FDG avid uptake in multiple sites. FNAC from right submandibular swelling and biopsy from left supraclavicular lymph node reported different pathology and to this FDG-PET scan added to the complexity and ambiguity of the case by reporting maximum FDG uptake in ileocaecal junction. Awareness of the occurrence of malignancy and its co-existence with extrapulmonary tuberculosis with different presentation and reliability of PET scan in such scenario should be kept in mind while making a precise diagnosis and scheduling appropriate management. 


\section{REFERENCES}

1. Sharma SK and Mohan A. Extrapulmonary tuberculosis. A review article. Indian J Med Res 2004; 120: 316-353.

2. Gnepp DR. Malignant mixed tumors of the salivary glands: a review. Pathol Annu 1993; 28(Pt 1):279-328.

3. Kato $\mathrm{H}$, Kanematsu M, Mizuta $\mathrm{K}$, Ito $\mathrm{Y}$ and Hirosec $\mathrm{Y}$. Carcinoma ex pleomorphic adenoma of the parotid gland: radiologic-pathologic correlation with MR imaging including diffusion-weighted imaging. AJNR Am J Neuroradiol 2008; 29:865-867

4. Waldron CA, el-Mofty SK and Gnepp DR. Tumors of the intraoral minor salivary glands: a demographic and histologic study of 426 cases. Oral Surg Oral Med Oral Pathol 1988; 66:323-333.

5. Furukawa M, Suzuki H, MatsuuraK, etal. Carcinoma ex pleomorphic adenoma of the palatal minor salivary gland with extension into the nasopharynx. Auris Nasus Larynx 2001; 28:279-281.

6. Kaplan $\mathrm{MH}$, Armstrong $\mathrm{D}$ and Rosen P. Tuberculosis complicating neoplastic disease. A review of 201 cases. Cancer 1974; 33:850-858.

7. Falagas ME, Kouranos VD, Athanassa Z and Opterides PK. Tuberculosis and malignancy. Q J Med 2010;103:461-487.

8. Al Bisher $\mathrm{H}$. A rare case of primary tuberculosis infection with concurrent pleomorphic adenoma of the parotid gland. Infectious Disease Reports 2010; 2:6.

Authors Contribution:

MS - Contribution- Data collection and review of literature; NL - Concept of study and surgical treatment of patient; SG - Design of the study, data collection and concept of the study; SS - Radiation treatment for the patient will come here; GG - Histopathological reporting of patient; SD - Radiation treatment for the patient.

Source of Support: Nil. Conflict of Interest: None. 